\title{
Ageing, urban marginality, and health in Ghana
}

Dominic A. Alaazi ${ }^{1}$, Devidas Menon ${ }^{1}$, Tania Stafinski ${ }^{1}$, Gian Jhangri ${ }^{1}$, Joshua Evans ${ }^{2}$, Stephen Hodgins $^{1}$

${ }^{1}$ School of Public Health, University of Alberta, 3-300 Edmonton Clinic Health Academy, 11405 87 Ave, Edmonton, Alberta, T6G 1C9, Canada.

${ }^{2}$ Earth and Atmospheric Sciences, University of Alberta, Tory Building 3-123, Edmonton, Alberta, T6G 2 E3.

\begin{abstract}
The world's population is rapidly ageing. Global estimates for the next three decades indicate a two-fold increase in the population of older adults aged $\geq 60$ years. Nearly $80 \%$ of this growth will occur in low and middle-income countries in Asia and sub-Saharan Africa, where population health is already under threat from poverty, degraded environments, and deficient healthcare systems. Although the world's poorest region, sub-Saharan Africa, ironically, will witness the fastest growth in older populations, rising by $64 \%$ over the next 15 years. Indications are that the majority of this population will live in resource-poor settings, characterized by deficient housing and neighbourhood conditions. Yet, very little research has systematically examined the health and wellbeing of older adults in such settings. Drawing on the ecological theory of ageing, the present study explores the living conditions and quality of life of elderly slum dwellers in Ghana, a sub-Saharan African country with a growing population of older adults. Data collection was undertaken in two phases in two environmentally contrasting neighbourhoods in Accra, Ghana. In Phase 1, we carried out a cross-sectional survey of older adults in a slum community $(n=302)$ and a non-slum neighbourhood $(n=301)$, using the World Health Organization quality of life assessment tool (WHOQoL-BREF). The survey data were complemented in Phase 2 with qualitative interviews involving a sample of community dwelling older adults $(N=30)$, health service providers $(N=5)$, community leaders $(N=2)$, and policymakers $(\mathrm{N}=5)$. Preliminary analysis of the survey data revealed statistically significant differences in the social and environment domains of quality of life, while the qualitative data identified multiple health barriers and facilitators in the two neighbourhoods. Insights from the research are expected to inform health and social interventions for older slum dwellers in Ghana.
\end{abstract}

\section{Key words}

Ghana, Health, Older adults, Quality of life, Urban slums

Cite as: Alaazi DA, Menon D, Stafinski T, Jhangri G, Evans J, Hodgins S. 2019. Ageing, urban marginality, and health in Ghana. Alberta Academic Review, Vol 2 (3) 11, CASCH Special Issue (not peer-reviewed), DOI: 10.29173/aar102. 